\title{
Oral Administration of Mifepristone Combined with Ultrasound- Guided Radiofrequency Ablation in Treating Patients with Uterine Fibroids: Efficacy, Safety, and Alternations of Inflammatory Cytokines, Adhesion Molecules, and Growth Factors
}

\author{
Aiqin Hou, Zhen Yan, Yuanyuan Zhang, and Jing Hou \\ Affiliated Hospital of Yan'an University, China \\ Correspondence should be addressed to Jing Hou; hgfytu2233@163.com
}

Received 25 March 2021; Revised 8 April 2021; Accepted 12 April 2021; Published 17 May 2021

Academic Editor: Songwen Tan

Copyright (c) 2021 Aiqin Hou et al. This is an open access article distributed under the Creative Commons Attribution License, which permits unrestricted use, distribution, and reproduction in any medium, provided the original work is properly cited.

Uterine fibroids are one of the most common and yet understudied diseases in women mainly during their reproductive years. The study analyzed the efficacy and safety of oral administration of mifepristone combined with ultrasound-guided radiofrequency ablation in treating patients with uterine fibroids, as well as changes of inflammatory cytokines including procalcitonin (PCT), high-sensitivity C-reactive protein (hs-CRP), and tumor necrosis factor-alpha (TNF- $\alpha$ ), adhesion molecules including monocyte chemotactic protein 1 (MCP-1) and soluble intercellular adhesion molecule 1 (sICAM-1), and growth factors including vascular endothelial growth factor (VEGF), epidermal growth factor (EGF), basic fibroblast growth factor (bFGF), transforming growth factor- $\beta$ (TGF- $\beta$ ), and TGF- $\beta$ receptor. A total of 130 patients who were admitted into our hospital for uterine fibroids from December 2016 to June 2018 were included, among which 65 women were given ultrasound-guided radiofrequency ablation alone (control group) and the remaining women were given oral administration of mifepristone combined with ultrasoundguided radiofrequency ablation (experimental group). Reduced volumes of uterus and fibroids were observed in both two groups after treatment $(P<0.05)$. It was found that the experimental group exhibited higher total effective rate and smaller volumes of uterus and fibroids than the control group $(P<0.05)$. Following treatment, the levels of MCP-1, sICAM-1, VEGF, EGF, bFGF, TGF- $\beta$, TGF- $\beta$ receptor, PCT, hs-CRP, and TNF- $\alpha$ in the serum in the two groups were declined $(P<0.05)$, while this decline was more significantly in the experimental group than the control group $(P<0.05)$. Besides, there was no significant difference in the incidence rate of adverse reactions between the two groups $\left(\chi^{2}=0.781, P>0.05\right)$. These results collectively indicate the high efficacy and safety of oral administration of mifepristone combined with ultrasound-guided radiofrequency ablation in treating patients with uterine fibroids, as this combination therapy could inhibit the expressions of inflammatory cytokines, adhesion molecules, and growth factors.

\section{Introduction}

Uterine fibroids are commonly seen in benign uterine tumors in fertile females, also defined as fibroids or myomas $[1,2]$. It influences on extensive population and the estimated prevalence may range from 80 to $90 \%$ by the age of 50 years [3]. Some research found that it occurs more often among older female and African descent that is likely to be caused by the effect of both genetic and environmental factors [4].
Although many females with fibroids are symptom-free, nearly $30 \%$ of them reveal some symptoms based on different location and size, involving back pain, pelvic pain and pressure, urinary frequency and urgency, anemia, constipation, abnormal uterine bleeding, and infertility $[5,6]$. The current available approach for uterine fibroids is mainly surgical interventions, but the strategy of treatment is decided by a variety of factors, including patient's age, delivery time, desire to remain fertility or rejection of radical therapy such as 
hysterectomy, also associating with fibroid's location, size and quantity, and the severity of symptoms $[7,8]$. More proof demonstrates that the economic impact of surgical interventions is huge, and new effective treatments for uterine fibroids are necessity, especially for a female who wants to retain their fertility [9].

Medication combined with nonsurgical therapy may be an actionable alternative for uterine fibroid, and over the last decade, the application of selective progesterone receptor modulators called as antiprogestins have been prevailing [10]. Mifepristone, one kind of progesterone receptors inhibitors, was the first effective compound in the use of long-term treatment of fibroids, since fibroid growth decided by the sexual steroids, and more study indicated that mifepristone can produce unique endometrial changes and decrease the growth of uterine fibroids through a variety of ways [1012]. Except for medication interruption, ultrasound-guided radiofrequency ablation (RFA) was performed in a minimally invasive manner, which is widely accepted as a reliable and effective treatment. It can induce coagulation necrosis by entering uterine fibroids through laparoscopy, vagina, or cervix [13].

Cell adhesion molecules (CAMs) possess a family of molecules that mediate the contact and binding between cells or between cells and extracellular matrix. Adhesion molecules play crucial roles in cell recognition, cell activation and signal transduction, cell proliferation and differentiation, and cell motility. They are also engaged in various biological processes, such as immune response, inflammation, coagulation, tumor metastasis, and wound healing [14, 15]. Growth factors are secreted by many cell types that could regulate cellular growth, proliferation, and differentiation. Uterine cellular events such as proliferation and differentiation are regulated by activation of growth factors acting by paracrine or autocrine mechanisms [16]. Interestingly, the presence of inflammatory cells in uterine fibroids may result in excessive extracellular matrix (ECM) production and tissue remodeling, eventually leading to leiomyoma growth [17]. Therefore, in this study, the changes of serum inflammatory cytokines including inflammatory cytokines including procalcitonin (PCT), high-sensitivity C-reactive protein (hs-CRP), and tumor necrosis factor-alpha (TNF- $\alpha$ ); adhesion molecules including monocyte chemotactic protein 1 (MCP-1) and soluble intercellular adhesion molecule 1 (sICAM-1); and growth factors including vascular endothelial growth factor (VEGF), epidermal growth factor (EGF), basic fibroblast growth factor (bFGF), transforming growth factor- $\beta$ (TGF$\beta$ ), and TGF- $\beta$ receptor will be analyzed by mifepristone combined with ultrasound-guided radiofrequency ablation, in order to provide more reliable data for clinical management for uterine fibroid.

\section{Materials and Methods}

2.1. Patient Enrollment. In this study, we recruited 130 patients who were diagnosed with uterine fibroids in our hospital from December 2016 to June 2018. All patients could provide complete clinical data. Eligible study subjects should meet the following inclusion criteria: presence of symptoms, such as anemia, abdominal pain, and menorrhagia; diagnosed as submucosal uterine fibroids in accordance with the International Federation of Gynecology and Obstetrics (FIGO) classification I-II [18]; clear presentation of size and location of fibroids through transabdominal ultrasound, with a safe access path; without undergoing high-intensity focused ultrasound (HIFU) or surgical resection before ultrasound guided radiofrequency ablation; and signing an informed consent approved by the Ethics Committee of our hospital. Patients were excluded from the study if they were complicated by other malignant tumors, severe liver and kidney dysfunction, severe metabolic system diseases, and severe neurological diseases, without complete clinical data, during menstruation, pregnancy or lactation, with acute pelvic inflammatory disease, allergic to drugs mentioned in this study, and had taken hormone drugs in recent two months.

2.2. Patient Information. Among 130 patients with uterine fibroids, there were 75 cases presenting abdominal pain, 53 presenting secondary anemia, 103 cases presenting menorrhagia, 18 cases presenting difficult defecation, 32 cases presenting frequent urination, and 53 cases presenting other symptoms. These 130 patients were divided into the control group $(n=65)$ and the experimental group $(n=65)$ according to the treatment method. The patients in the control group were aged ranging from 29 to 35 years, with an average of age $(32.3 \pm 3.6)$ years, and the disease duration was 1 to 4 years with an average of disease duration $(2.4 \pm 1.3)$ years. The control group had an average of fibroid diameter $(3.32 \pm 0.98) \mathrm{cm}$, with 23 cases of single fibroid and 42 cases of multiple fibroids. The patients in the experimental group were aged ranging from 29 to 34 years, with an average of age $(32.4 \pm 3.8)$ years, and the disease duration was 1 to 4 years with an average of disease duration $(2.4 \pm 1.5)$ years. The experimental group had an average of fibroid diameter $(3.19 \pm 1.08) \mathrm{cm}$, with 26 cases of single fibroid and 39 cases of multiple fibroids. There were 40 cases with birth history and 8 cases with a history of induced abortion in the control group and 34 cases with birth history and 12 cases with a history of induced abortion. There was no statistical difference in age, disease duration, diameter of fibroids, single or multiple fibroids, birth history, and history of induced abortion between the two groups $(P>0.05)$.

2.3. Treatment. Ultrasound-guided radiofrequency ablation was performed. The patients were treated with routine vaginal and cervical disinfection at first, and the curettage was performed after the fibroid was detected by the intraluminal probe and abdominal probe. If the diameter of the myoma was less than $3 \mathrm{~cm}$, the self-coagulation knife was directly placed into the center of the myoma. If the diameter of the myoma is more than $3 \mathrm{~cm}$, the self-coagulation knife was penetrated into from one side to the other side. With the guide of ultrasound, self-coagulation knife radiofrequency with a power of $30+3 \mathrm{~W}$ was leaded into lesions longitudinally and withdrawn when reaching full electrical resistance in tissue, keeping the process for many times until all lesions were coagulated and necrotic. The patients were given hemostatics or antibiotics to prevent bleeding and infection after 
operation. The average operation time was $(27.43 \pm 5.98)$ min, and the average blood loss was $(14.2 \pm 4.8) \mathrm{ml}$. The patients in the experimental group continued to receive oral administration of mifepristone $(10 \mathrm{mg} /$ time, once a day for 3 months).

\subsection{Collection and Processing of Human Blood Sample. Blood} samples were collected from all patients before treatment and 3 months after treatment, respectively, and two test tubes of samples were collected at one time for enzyme-linked immunosorbent assay (ELISA) of adhesion molecules, growth factors, and inflammatory factors. The collection processes were as followed: elbow venous blood $(5 \mathrm{ml})$ was collected from each patient after fasting for at least 8 hours and then stored in refrigerator at $4^{\circ} \mathrm{C}$ for 45 minutes. The blood sample was centrifuged for $15 \mathrm{~min}$ at $3500 \mathrm{r} / \mathrm{min}$ in a centrifuge with $10 \mathrm{~cm}$ radius to get the supernatant as serum. The serum was immediately stored at $-80^{\circ} \mathrm{C}$.

2.5. Experimental Instruments. Experimental instruments used in this study were as follows: automatic multifunction microplate reader (Fluostar Omega, Bio-Gene Technology Co., Ltd. China), Panasonic refrigerator (MDF-U5412, SANYO Electric Co., Ltd. Japan), spectrophotometer (OD1000+, Shanghai Gene Sci Medical Technology Co., Ltd., China), automatic biochemical analyzer (AU64, Olympus Corporation. J.P.), and ELISA kits (Sigma-Aldrich. USA).

2.6. ELISA. The blank control, sample, and standard wells were set. At first, $50 \mu \mathrm{l}$ serum sample was put into the standard holes; $40 \mu \mathrm{l}$ of the PH9 buffer with $0.05 \mathrm{~mol} / \mathrm{L}$ concentration and $10 \mu \mathrm{l}$ serum samples were added into sample holes successively, then mixing them gently. The reaction holes covered by microplate sealers placed at $37^{\circ} \mathrm{C}$ for 30 minutes, then pouring off the sample liquid completely and cleaning the holes 3 times with butter diluted by distilled water, 3 minutes each time. With the exception of blank holes, $50 \mu \mathrm{l}$ of ELISA reagent was added into the other holes; then, $\mathrm{A}$ and $\mathrm{B}$ color reagent with $50 \mu \mathrm{l}$ was poured into the three kinds of holes successively and gently mix the liquid in each hole. Adding $50 \mu \mathrm{l}$ of reaction termination solution to each hole until color yellow appears, and the blank holes were used as the zero reference value within 15 minutes, the absorbance (OD value) in each hole was measured with a spectrophotometer with $450 \mathrm{~nm}$ wavelength, and the concentrations of VEGF, EGF, bFGF, TGF $\beta$, MCP-1, sICAM1 , PCT, hs CRP, and TNF- $\alpha$ in the sample were calculated by using the standard curve.

2.7. Efficacy Evaluation. Clinical response of patients with uterine fibroids to ultrasound-guided radiofrequency ablation alone or combined with oral administration of mifepristone was evaluated. Complete response was defined when all symptoms and signs of the patients disappeared, and uterine fibroids have found to be disappeared in B-ultrasound examination of uterine appendages. Excellent response was defined when the symptoms and signs of the patients basically disappeared or significantly reduced, and the volume reduction rate of uterine fibroids was more than $50 \%$ indicated in B-ultrasound examination of uterine appendages.
Good response was defined when the patient's symptoms and signs have been alleviated, and the volume of uterine fibroids reduced by $25 \%-50 \%$ showed in B-ultrasound examination of uterine appendages. No response was defined when there was no any improvement in symptoms, and Bultrasound examination of uterine appendages described that the volume of uterine fibroids decreased less than $25 \%$ or increased. Total response rate $=100.00 \%$ - rate of case with no response.

2.8. Calculation of Volumes of Uterine and Fibroids. The volumes of uterus and fibroids were calculated following the formula: $V=\pi a b c / 6$, where $a b c$ indicate the length, width, and thickness of uterus and fibroids, respectively.

2.9. Data Analysis. All data were processed by the SPSS 20.0 software. The measurement data were defined as mean \pm standard deviation and analyzed by the $t$-test. The counting data were described by ratio or percentage and analyzed by the chi-square test. A level of $P<0.05$ was considered statistically significant.

\section{Result}

3.1. Oral Administration of Mifepristone Combined with Ultrasound-Guided Radiofrequency Ablation Showed Favorable Patient Outcome. There were 29 cases showing complete response, 22 cases showing excellent response, 12 cases showing good response, and 2 cases showing no response in the experimental group. There were 19 cases showing complete response, 25 cases showing excellent response, 7 cases showing good response, and 14 cases showing no response in the control group. The total response rate in the experimental group was higher than that in control group $\left(\chi^{2}=7.195, P<0.05\right.$, Table 1$)$. Next, the volumes of uterus and fibroids were measured and calculated. It was found that patients with uterine fibroids exhibited reduced volumes of uterus and fibroids following either ultrasound guided radiofrequency ablation or oral administration of mifepristone combined with ultrasound guided radiofrequency ablation $(P<0.05)$. At the same time, the volumes of uterus and fibroids were smaller in the experimental group than those in the control group $(P<0.05$, Table 2$)$.

3.2. Oral Administration of Mifepristone Combined with Ultrasound-Guided Radiofrequency Ablation Notably Reduced the Secretion of Adhesion Molecules. Adhesion molecules represent a group of cell-surface molecules and are likely to be of central importance in mediating cell-extracellular matrix and specific cell-cell interactions within both neoplastic and inflammatory sites. MCP-1 and sICAM-1 expressions were associated with the pathogenesis of endometriosis from previous evidence. We are interested in a hypothesis that MCP-1 and SICAM-1 expressions are altered in patients with uterine fibroids undergoing oral administration of mifepristone combined with ultrasound-guided radiofrequency ablation. We found that the serum levels of MCP-1 and sICAM-1 in patients with uterine fibroids were significantly decreased following either ultrasound-guided radiofrequency ablation or oral administration of mifepristone combined with 
TABLE 1: Clinical response of patients with uterine fibroids to ultrasound-guided radiofrequency ablation alone or combined with oral administration of mifepristone.

\begin{tabular}{lcccccc}
\hline Group & $\begin{array}{c}\text { Complete response } \\
{[n(\%)]}\end{array}$ & $\begin{array}{c}\text { Excellent response } \\
{[n(\%)]}\end{array}$ & $\begin{array}{c}\text { Good response } \\
{[n(\%)]}\end{array}$ & No response $[n(\%)]$ & Total response rate & $\chi$ \\
\hline Experimental group & $29(44.62 \%)$ & $22(33.85 \%)$ & $12(18.46 \%)$ & $2(3.08 \%)$ & $96.92 \%$ & 7.195 \\
Control group & $19(29.23 \%)$ & $25(38.46 \%)$ & $7(10.77 \%)$ & $14(21.54 \%)$ & $78.46 \%$ & $<05$ \\
\hline
\end{tabular}

TABLE 2: The volumes of uterus and fibroids of patients with uterine fibroids following ultrasound-guided radiofrequency ablation alone or combined with oral administration of mifepristone.

\begin{tabular}{|c|c|c|c|c|}
\hline Group & $N$ & Before or after treatment & The volume of uterus $\left(\mathrm{cm}^{3}\right)$ & The volumes of fibroids $\left(\mathrm{cm}^{3}\right)$ \\
\hline \multirow{2}{*}{ Control group } & \multirow{2}{*}{65} & Before & $244.43 \pm 25.65$ & $77.70 \pm 7.33$ \\
\hline & & After & $188.34 \pm 19.44$ & $55.32 \pm 8.60$ \\
\hline$t$ & \multirow{4}{*}{65} & & 14.658 & 26.443 \\
\hline$P$ & & & 0.001 & 0.001 \\
\hline \multirow{2}{*}{ Experimental group } & & Before & $245.32 \pm 30.22$ & $78.16 \pm 8.54$ \\
\hline & & After & $160.54 \pm 10.55^{*}$ & $41.54 \pm 5.87^{*}$ \\
\hline$t$ & & & 32.443 & 38.667 \\
\hline$P$ & & & 0.001 & 0.001 \\
\hline
\end{tabular}

Compared to the control group, ${ }^{*} P<0.05$.

ultrasound-guided radiofrequency ablation $(P<0.05)$. Besides, the serum levels of MCP-1 and sICAM-1 were declined in the experimental group compared with the control group $(P<0.05$, Table 3$)$.

3.3. Oral Administration of Mifepristone Combined with Ultrasound-Guided Radiofrequency Ablation Remarkably Reduced the Release of Growth Factors. Growth factors, relatively small and stable, secreted, or membrane-bound polypeptide ligands, have been implicated in proliferation, differentiation, angiogenesis, survival, inflammation, and tissue repair, or fibrosis through the activation of signal transduction pathways by binding to their receptors on the surface of target cells. Numerous differentially expressed growth factors have been identified in leiomyoma and myometrial cells. Given the roles of growth factors in the pathogenesis of uterine fibroids, we are wondering the effects of oral administration of mifepristone combined with ultrasound-guided radiofrequency ablation on the release of growth factors in patients with uterine fibroids. Results revealed that the serum levels of VEGF, EGF, bFGF, TGF- $\beta$, and TGF- $\beta$ receptor in patients with uterine fibroids were significantly decreased following either ultrasound-guided radiofrequency ablation or oral administration of mifepristone combined with ultrasound-guided radiofrequency ablation $(P<0.05)$. Besides, the serum levels of VEGF, EGF, $\mathrm{bFGF}, \mathrm{TGF}-\beta$, and TGF- $\beta$ receptor were declined in the experimental group compared with the control group $(P<0.05$, Table 4$)$

3.4. Oral Administration of Mifepristone Combined with Ultrasound-Guided Radiofrequency Ablation Significantly Inhibited Inflammatory Cytokine Production. Inflammatory imbalance has been demonstrated to contribute to leio- myoma development and growth, which would provide an avenue for the development of preventative treatments. In this part, we attempted to examine inflammatory response in patients with uterine fibroids after oral administration of mifepristone combined with ultrasound-guided radiofrequency ablation. After treatment, the serum levels of PCT, hs-CRP, and TNF- $\alpha$ were declined in the experimental and control group $(P<0.05)$, and the experimental group exhibited lower serum levels of PCT, hs-CRP, and TNF- $\alpha$ than the control group $(P<0.05$, Table 5).

3.5. The Incidence of Adverse Reactions. There were 2 cases with nausea, 2 cases with mild dizziness, and 1 case with vaginal discharge in the control group, with a $7.69 \%$ incidence rate of adverse reactions. There were 3 cases of nausea, 2 cases of mild dizziness, and 1 case with vaginal discharge in the experimental group, with a $9.23 \%$ incidence rate of adverse reactions. There was no significant difference in the incidence of adverse reactions between the two groups $\left(\chi^{2}=0.781, P>0.05\right)$.

\section{Discussion}

The management of fibroids has been multidisciplinary in the past years. Different therapeutic approaches are now available for women with uterine fibroids, among which medical therapy may be less invasive [19]. Medical therapy is an option for women with symptomatic uterine fibroids who consider fertility preservation or expect a less aggressive operation after shrinkage of the uterine volume [20]. The underlying mechanism of most medical therapies is based on the inhibition of myoma growth. Recently, the USguided RF ablation is reported as a relatively safe and 
TABLE 3: The serum levels of MCP-1 and sICAM-1 in patients with uterine fibroids following ultrasound-guided radiofrequency ablation alone or combined with oral administration of mifepristone.

\begin{tabular}{|c|c|c|c|c|}
\hline Group & $N$ & Before or after treatment & MCP-1 $(\mu \mathrm{g} / \mathrm{L})$ & sICAM-1 $(\mu \mathrm{g} / \mathrm{L})$ \\
\hline \multirow{2}{*}{ Control group } & \multirow{2}{*}{65} & Before & $33.29 \pm 3.11$ & $458.77 \pm 29.12$ \\
\hline & & After & $25.48 \pm 6.22$ & $404.32 \pm 19.17$ \\
\hline$t$ & \multirow{4}{*}{65} & & 15.487 & 21.909 \\
\hline$P$ & & & 0.001 & 0.001 \\
\hline \multirow{2}{*}{ Experimental group } & & Before & $34.14 \pm 6.55$ & $459.32 \pm 17.12$ \\
\hline & & After & $19.78 \pm 4.08^{\#}$ & $366.56 \pm 12.54^{\#}$ \\
\hline$t$ & & & 19.221 & 33.762 \\
\hline$P$ & & & 0.001 & 0.001 \\
\hline
\end{tabular}

Compared to the control group, ${ }^{\#} P<0.05$; MCP-1: monocyte chemotactic protein 1; sICAM-1: soluble intercellular adhesion molecule 1.

TABLE 4: The serum levels of TGF- $\beta$, TGF- $\beta$ receptor, VEGF, EGF, and bFGF in patients with uterine fibroids following ultrasound-guided radiofrequency ablation alone or combined with oral administration of mifepristone.

\begin{tabular}{|c|c|c|c|c|c|c|c|}
\hline Group & $N$ & Before or after treatment & TGF- $\beta(\mu \mathrm{g} / \mathrm{L})$ & TGF- $\beta$ receptor $(\mu \mathrm{g} / \mathrm{L})$ & VEGF (ng/L) & EGF (ng/L) & bFGF (ng/L) \\
\hline \multirow{2}{*}{ Control group } & \multirow{2}{*}{65} & Before & $45.67 \pm 8.12$ & $25.32 \pm 4.66$ & $105.38 \pm 35.11$ & $12.54 \pm 3.42$ & $103.84 \pm 21.74$ \\
\hline & & After & $39.40 \pm 7.77$ & $20.67 \pm 3.12$ & $89.24 \pm 20.26$ & $9.08 \pm 2.17$ & $81.95 \pm 13.82$ \\
\hline$t$ & & & 8.324 & 6.521 & 14.183 & 6.196 & 16.412 \\
\hline$P$ & & & 0.001 & 0.001 & 0.001 & 0.001 & 0.001 \\
\hline \multirow{2}{*}{ Experimental group } & \multirow{2}{*}{65} & Before & $46.14 \pm 8.45$ & $26.91 \pm 6.33$ & $106.54 \pm 34.97$ & $12.87 \pm 4.15$ & $104.67 \pm 19.83$ \\
\hline & & After & $30.33 \pm 9.56^{8}$ & $15.73 \pm 5.38^{\&}$ & $71.54 \pm 12.93^{8}$ & $5.01 \pm 0.87^{\&}$ & $52.71 \pm 13.26^{8}$ \\
\hline$t$ & & & 21.435 & 11.548 & 37.548 & 7.955 & 14.084 \\
\hline$P$ & & & 0.001 & 0.001 & 0.001 & 0.001 & 0.001 \\
\hline
\end{tabular}

Compared to the control group, ${ }^{8} P<0.05$; TGF- $\beta$ : transforming growth factor $\beta$; VEGF: vascular endothelial growth factor; EGF: epidermal growth factor; bFGF: basic fibroblast growth factor.

TABLE 5: The serum levels of PCT, hs-CRP, and TNF- $\alpha$ in patients with uterine fibroids following ultrasound-guided radiofrequency ablation alone or combined with oral administration of mifepristone.

\begin{tabular}{|c|c|c|c|c|c|}
\hline Group & $N$ & Before or after treatment & PCT $(\mathrm{pg} / \mathrm{ml})$ & hs-CRP (pg/ml) & TNF- $\alpha(\mathrm{pg} / \mathrm{ml})$ \\
\hline \multirow{2}{*}{ Control group } & \multirow{2}{*}{65} & Before & $135.21 \pm 57.44$ & $221.02 \pm 48.15$ & $324.96 \pm 38.75$ \\
\hline & & After & $92.13 \pm 16.77$ & $182.59 \pm 41.87$ & $254.42 \pm 47.36$ \\
\hline$t$ & \multirow{4}{*}{65} & & 37.952 & 27.841 & 33.149 \\
\hline$P$ & & & 0.001 & 0.001 & 0.001 \\
\hline \multirow{2}{*}{ Experimental group } & & Before & $134.84 \pm 56.81$ & $222.43 \pm 49.01$ & $327.15 \pm 39.74$ \\
\hline & & After & $56.34 \pm 17.33^{\$}$ & $103.46 \pm 33.67^{\$}$ & $201.26 \pm 31.28^{\$}$ \\
\hline$t$ & & & 45.529 & 47.586 & 44.437 \\
\hline$P$ & & & 0.001 & 0.001 & 0.001 \\
\hline
\end{tabular}

Compared to the control group, ${ }^{\&} P<0.05$; PCT: procalcitonin; hs-CRP: high-sensitivity C-reactive protein; TNF- $\alpha$ : tumor necrosis factor- $\alpha$.

effective procedure for uterine fibroids with various localizations and sizes [21]. In this study, we explored the clinical efficacy of medical therapy (mifepristone) combined with the US-guided RF ablation in treating women with uterine fibroids and the effects of his combined approach on serum levels of MCP-1, sICAM-1, and TGF- $\beta$ of patients.

In 2008, Esteve et al. [22] performed a randomized controlled trial by randomly assigning 100 women to receive oral mifepristone $5 \mathrm{mg}$ or $10 \mathrm{mg}$ daily for 3 months and found $5 \mathrm{mg}$ doses of mifepristone reduced leiomyoma and uterine volumes similar to $10 \mathrm{mg}$ doses. In 2009, Engman et al. [23] randomized 30 women with uterine fibroids to receive either $50 \mathrm{mg}$ mifepristone or placebo every other day during 3 months prior to surgery and evaluated uterine blood flow and leiomyoma volume once a month until surgery. It was revealed mifepristone may offer an effective treatment option for women with uterine fibroids with uterovaginal bleeding. As shown in our study, compared with women with uterine fibroids receiving the US-guided RF ablation alone, women with uterine fibroids receiving the US-guided RF ablation 
and administration of $10 \mathrm{mg}$ mifepristone once a day for 3 months had fewer volumes of uterus and fibroids.

Another important finding of the study is that the USguided RF ablation and administration of $10 \mathrm{mg}$ mifepristone more significantly reduced serum levels of MCP-1, sICAM-1, and TGF- $\beta$ than ablative technology alone. Higher production of MCP-1 is associated with augmented inflammatory reaction in endometrium, which might be detrimental to reproductive outcome in women with uterine fibroids [24]. CAMs have been implicated in leukocyte-endothelial interactions, including ICAM-1, vascular cell adhesion molecule-1 (VCAM-1), and E-selectin all of which are regulated by sex steroids, while CAM expression in the uterine and ovarian appears to be associated with certain disease states in women of reproductive age [25]. The normal cyclic variation in peripheral sICAM-1 and sVCAM-1 levels may reflect uterine and/or ovarian tissue remodeling events, and sICAM-1 levels could be utilized as biological markers of endometriosis [26-28]. Numerous differentially expressed growth factors have been identified in leiomyoma and myometrial cells. $\mathrm{Mu}$ et al. [29] and Plewka et al. [30] both demonstrated high expression of VEGF that can probably cause malignant transformation and more extensive growth of leiomyoma. EGF stimulates DNA synthesis and polyploidization in leiomyomal smooth muscle cells and participates in the pathogenesis of human uterine fibroids [31]. Previous evidence showed that the regulation of bFGF in leiomyomas and in myometrium is affected by sex steroid hormones [32]. TGF- $\beta$ is a polypeptide that consists of three isoforms, TGF- $\beta 1$, TGF- $\beta 2$, and TGF- $\beta 3$. At present, TGF- $\beta$ is considered to be one of the key factors in the pathophysiology of uterine fibroids [33]. TGF- $\beta$ expression was increased as the growth of uterine fibroids in women bearing childbearing age, which shows considerable potential as a therapeutic target for uterine fibroids [34]. Chronic inflammation is regarded as one of the challenges for the treatment of human diseases [35]. Inflammatory imbalance has been demonstrated to contribute to leiomyoma development and growth, which would provide an avenue for the development of preventative treatments. As reported in the study performed by Sevostyanova et al. [36], activation of adaptive immunity, angiogenic factors, and inflammatory cell reactions was observed in patients with uterine fibroids with reproductive failure, that is why detection of CRP, IFN- $\gamma$, and TNF- $\alpha$ in serum is necessary to perform in pregravid preparation of women, including in vitro fertilization program. Considering the implication of inflammatory cytokines, adhesion molecules, and growth factors in the development of uterine fibroids, their repression caused by the US-guided RF ablation and administration of $10 \mathrm{mg}$ mifepristone further demonstrated the clinical efficacy of mifepristone combined with the US-guided RF ablation in treating women with uterine fibroids.

In summary, our data support the higher clinical efficacy of mifepristone combined with the US-guided RF ablation than the US-guided RF ablation alone. Nevertheless, these results are preliminary, as the number of patients was restricted and the actual follow-up time was limited. In the future, we will extend sample size power and perform multi- center study, so as to provide more evidence for clinical promotion of mifepristone combined with the US-guided RF ablation.

\section{Data Availability}

The data used to support the findings of this study are included within the article.

\section{Conflicts of Interest}

The authors declare that they have no conflict of interest.

\section{Authors' Contributions}

Aiqin Hou and Zhen Yan contributed equally to this work.

\section{Acknowledgments}

The study is supported by Yan'an University Cultivation Fund Project (2018ZD-02), Basic research program of Natural Science in Shaanxi Province (2019JQ-986), and Yan'an science and Technology Bureau [(2020)64].

\section{References}

[1] M. Gupta, N. Jamwal, S. Sabharwal, and S. Sobti, "A prospective interventional study to evaluate the effects of medical therapy (Mifepristone $25 \mathrm{mg}$ ) on the management of uterine fibroids," Journal of Family Medicine and Primary Care, vol. 9, no. 7, pp. 3230-3235, 2020.

[2] A. S. Boosz, P. Reimer, M. Matzko, T. Romer, and A. Muller, "The conservative and interventional treatment of fibroids," Deutsches Ärzteblatt International, vol. 111, no. 51-52, pp. 877-883, 2014.

[3] M. Grube, F. Neis, S. Y. Brucker et al., "Uterine fibroids - current trends and strategies," Surgical Technology International, vol. 34, pp. 257-263, 2019.

[4] M. S. De La Cruz and E. M. Buchanan, "Uterine fibroids: diagnosis and treatment," American Family Physician, vol. 95, no. 2, pp. 100-107, 2017.

[5] E. Giuliani, S. As-Sanie, and E. E. Marsh, "Epidemiology and management of uterine fibroids," International Journal of Gynaecology and Obstetrics, vol. 149, no. 1, pp. 3-9, 2020.

[6] W. H. Parker, "Etiology, symptomatology, and diagnosis of uterine myomas," Fertility and Sterility, vol. 87, no. 4, pp. 725-736, 2007.

[7] F. Faustino, M. Martinho, J. Reis, and F. Aguas, "Update on medical treatment of uterine fibroids," European Journal of Obstetrics, Gynecology, and Reproductive Biology, vol. 216, pp. 61-68, 2017.

[8] J. Donnez and M. M. Dolmans, "Uterine fibroid management: from the present to the future," Human Reproduction Update, vol. 22, no. 6, pp. 665-686, 2016.

[9] J. Donnez, P. Arriagada, O. Donnez, and M. M. Dolmans, "Emerging treatment options for uterine fibroids," Expert Opinion on Emerging Drugs, vol. 23, no. 1, pp. 17-23, 2018.

[10] M. Farris, C. Bastianelli, E. Rosato, I. Brosens, and G. Benagiano, "Uterine fibroids: an update on current and emerging medical treatment options," Therapeutics and Clinical Risk Management, vol. Volume 15, pp. 157-178, 2019. 
[11] S. Mukherjee and S. Chakraborty, "A study evaluating the effect of mifepristone (RU-486) for the treatment of leiomyomata uteri," Nigerian Medical Journal, vol. 52, no. 3, pp. 150-152, 2011.

[12] C. Malartic, O. Morel, G. Akerman, L. Tulpin, P. Desfeux, and E. Barranger, "La mifépristone dans la prise en charge des fibromes utérins," Gynécologie, Obstétrique \& Fertilité, vol. 36, no. 6, pp. 668-674, 2008.

[13] L. D. Bradley, R. P. Pasic, and L. E. Miller, "Clinical performance of radiofrequency ablation for treatment of uterine fibroids: systematic review and meta-analysis of prospective studies," Journal of Laparoendoscopic \& Advanced Surgical Techniques. Part A, vol. 29, no. 12, pp. 1507-1517, 2019.

[14] A. Makker, M. M. Goel, D. Nigam et al., "Endometrial expression of homeobox genes and cell adhesion molecules in infertile women with intramural fibroids during window of implantation," Reproductive Sciences, vol. 24, no. 3, pp. 435444, 2017.

[15] M. S. Islam, A. Ciavattini, F. Petraglia, M. Castellucci, and P. Ciarmela, "Extracellular matrix in uterine leiomyoma pathogenesis: a potential target for future therapeutics," Human Reproduction Update, vol. 24, no. 1, pp. 59-85, 2018.

[16] P. Ciarmela, M. S. Islam, F. M. Reis et al., "Growth factors and myometrium: biological effects in uterine fibroid and possible clinical implications," Human Reproduction Update, vol. 17, no. 6, pp. 772-790, 2011.

[17] O. Protic, P. Toti, M. S. Islam et al., "Possible involvement of inflammatory/reparative processes in the development of uterine fibroids," Cell and Tissue Research, vol. 364, no. 2, pp. 415427, 2016.

[18] C. Perrine, M. Levaillant Jean, and F. Hervé, "Surgical techniques and outcome in the management of submucous fibroids," Current Opinion in Obstetrics \& Gynecology, vol. 25, pp. 332-338, 2013.

[19] S. K. Laughlin-Tommaso, "Non-surgical management of myomas," Journal of Minimally Invasive Gynecology, vol. 25, no. 2, pp. 229-236, 2018.

[20] M. H. Cheng, H. T. Chao, and P. H. Wang, "Medical treatment for uterine myomas," Taiwanese Journal of Obstetrics \& Gynecology, vol. 47, no. 1, pp. 18-23, 2008.

[21] G. Yuce, A. Tayarer, H. L. Keskin, B. Genc, and M. Canyigit, "Ultrasound guided percutaneous radiofrequency thermal ablation of symptomatic uterine fibroids - results from a single center and 52 weeks of follow up," Ginekologia Polska, vol. 91, no. 8, pp. 447-452, 2020.

[22] J. L. Carbonell Esteve, R. Acosta, B. Heredia, Y. Pérez, M. C. Y. Castañeda, and A. V. Hernández, "Mifepristone for the treatment of uterine leiomyomas: a randomized controlled trial," Obstetrics and Gynecology, vol. 112, no. 5, pp. 1029-1036, 2008.

[23] M. Engman, S. Granberg, A. R. Williams, C. X. Meng, P. G. Lalitkumar, and K. Gemzell-Danielsson, "Mifepristone for treatment of uterine leiomyoma. A prospective randomized placebo controlled trial," Human Reproduction, vol. 24, no. 8, pp. 1870-1879, 2009.

[24] S. Miura, K. N. Khan, M. Kitajima et al., "Differential infiltration of macrophages and prostaglandin production by different uterine leiomyomas," Human Reproduction, vol. 21, no. 10, pp. 2545-2554, 2006.

[25] M. Ueda, Y. Yamashita, M. Takehara et al., "Gene expression of adhesion molecules and matrix metalloproteinases in endo- metriosis," Gynecological Endocrinology, vol. 16, no. 5, pp. 391-402, 2002.

[26] L. Kuessel, R. Wenzl, K. Proestling et al., "Soluble VCAM1 soluble ICAM-1 ratio is a promising biomarker for diagnosing endometriosis," Human Reproduction, vol. 32, no. 4, pp. 110, 2017.

[27] N. Bonello and R. J. Norman, "Soluble adhesion molecules in serum throughout the menstrual cycle," Human Reproduction, vol. 17, no. 9, pp. 2272-2278, 2002.

[28] M. H. Wu, B. C. Yang, Y. C. Lee, P. L. Wu, and C. C. Hsu, "The differential expression of intercellular adhesion molecule-1 (ICAM-1) and regulation by interferon-gamma during the pathogenesis of endometriosis," American Journal of Reproductive Immunology, vol. 51, no. 5, pp. 373-380, 2004.

[29] Y. Mu, J. He, R. Yan, X. Hu, H. Liu, and Z. Hao, "IGF-1 and VEGF can be used as prognostic indicators for patients with uterine fibroids treated with uterine artery embolization," Experimental and Therapeutic Medicine, vol. 11, no. 2, pp. 645-649, 2016.

[30] D. Plewka, M. Morek, E. Bogunia, J. Waloszek, and A. Plewka, "Expression of VEGF isoforms and their receptors in uterine myomas," Ginekologia Polska, vol. 87, no. 3, pp. 166-177, 2016.

[31] Y. Y. Ren, H. Yin, R. J. Tian et al., "Different effects of epidermal growth factor on smooth muscle cells derived from human myometrium and from leiomyoma," Fertility and Sterility, vol. 96, no. 4, pp. 1015-1020.e1, 2011.

[32] X. Wu, A. Blanck, M. Olovsson, B. Möller, and B. Lindblom, "Expression of basic fibroblast growth factor (bFGF), FGF receptor 1 and FGF receptor 2 in uterine leiomyomas and myometrium during the menstrual cycle, after menopause and GnRHa treatment," Acta Obstetricia et Gynecologica Scandinavica, vol. 80, no. 6, pp. 497-504, 2001.

[33] M. Ciebiera, M. Wlodarczyk, M. Wrzosek et al., "Role of transforming growth factor $\beta$ in uterine fibroid biology," International Journal of Molecular Sciences, vol. 18, no. 11, p. 2435 , 2017.

[34] T. Shen, H. Shi, Q. Xu, Q. Song, Y. Xu, and Y. Huang, "Effects of TGF- $\beta$ on uterine fibroids of women of childbearing age and uterine artery embolization," Minimally Invasive Therapy \& Allied Technologies, vol. 26, no. 5, pp. 292-299, 2017.

[35] K. M. le, N. Trinh, V. D. Nguyen et al., "Investigating the AntiInflammatory Activity of Curcumin-Loaded Silica- Containing Redox Nanoparticles," Journal of Nanomaterials, vol. 2021, Article ID 6655375, 11 pages, 2021.

[36] O. Sevostyanova, T. Lisovskaya, G. Chistyakova et al., "Proinflammatory mediators and reproductive failure in women with uterine fibroids," Gynecological Endocrinology, vol. 36, no. sup1, pp. 33-35, 2020. 